\title{
Fibrous Hamartoma Of Infancy-Case Report.
}

\author{
Dr.Vishwapriya.M.Godkhindi ${ }^{1}$, Dr.Darshan.P.Meshram ${ }^{2,}$ Dr.Deshpande.S.A ${ }^{3}$, \\ Dr.Suvernekar.S. $V^{4}$. \\ 1 .Postgraduate resident; Department of Pathology,Dr.S.C.GMC.Nanded(MS),India. \\ 2 .Postgraduate resident; Department of Pathology,Dr.S.C.GMC.Nanded(MS),India. \\ 3.Professor and Head; Department of Pathology,Dr.S.C.GMC,Nanded(MS),India. \\ 4, Associate Professor; Department of Pathology,Dr.S.C.GMC,Nanded(MS),India.
}

\begin{abstract}
Fibrous hamartoma of infancy is an extremely rare benign proliferation of soft tissue of unknown histiogenesis, with less than 200 cases reported in medical literature, and commonly presenting as a rapidly growing solitary subcutaneous mass in an infant. More than $90 \%$ of the lesions occur during the $1^{\text {st }}$ year of life and approximately $25 \%$ being congenital. The lesion is twice as common in males and the common sites include axillae, chest wall, back, thigh, and the inguinoscrotal region.

We report the case of a 9 month old male child, born out of a non-consanguineous marriage and attaining normal developmental milestones, who presented with a rapidly growing right axillary mass of 5 months duration. The tumor was excised with wide surgical margins and the wound approximated with skin flap. The histopathological examination confirmed the diagnosis of Fibrous hamartoma of infancy.

We report this case because of its extreme rarity, uncanny appearance; a common mimicker of capricious childhood soft tissue neoplasms.
\end{abstract}

Keywords- Fibrous hamartoma of infancy, Childhood soft tissue neoplasms.

\section{Introduction}

Fibrous hamartoma of infancy is a rare benign fibrous proliferation of soft tissue of unknown histiogenesis. Till date less than 200 cases have been reported in medical literature. ${ }^{[1]}$ This rare entity was first described by Reye in 1956 and he called it 'subdermal fibrous tumor of childhood' ${ }^{[2]}$ Infancy. ${ }^{[3]}$

Enzinger in 1965 after a review of 30 cases nomenclatured the lesion as 'Fibrous Hamartoma of

This rare lesion is usually diagnosed during the first 2 years of life with greater than $91 \%$ of reported cases presenting during the first year of life, the lesion has been described to be congenital in a quarter of the cases. ${ }^{[4]}$ There is a distinctive male preponderance and the males being afflicted twice to thrice as more commonly over females. ${ }^{[5]}$

These lesions are solitary and present as a rapidly growing subcutaneous mass, most commonly over the axillae, arm, shoulder, chest wall and back. ${ }^{[6]}$ This lesion belong to a genre of their own and are quite liable to be misdiagnosed as a malignant soft tissue neoplasm of infancy like rhabdomyosarcoma and risk being overtreated with catastrophic results.

These tumors tread a benign path and are best treated by wide local exeresis with tissue reconstruction. This is to minimize the high chances of recurrence that is noted in $10-15 \%$ of the ill primed cases. $^{[4]}$

\section{Case Report}

We report the case of a 9 month old male infant born out of a non-consanguineous marriage and uneventful perinatal period, who presented with the complains of a rapidly progressive right axillary mass of 5 months duration.

Examination revealed a firm to hard solitary, non tender, mobile mass in the antero-medial aspect of right axilla of dimensions $5 \times 4 \times 4 \mathrm{cms}$. The lesion was in the subcutaneous plane with restricted arm movements with no attachment to the underlying muscle or bone. No neurological deficits or trophic skin changes noted. There were no similar masses anywhere over the body. No e/o any lymphadenopathy.

After complete hematological, biochemical and pre anaesthetic workup and with the informed written parental consent the child was posted for surgical exeresis reconstruction of the lesion.

Intraoperatively a firm to hard, unencapsulated mass with infiltrating margins was noted in the subcutaneous plane.

The tumor was excised taking wide surgical margins and the tissue defect reconstructed via skin flap. 


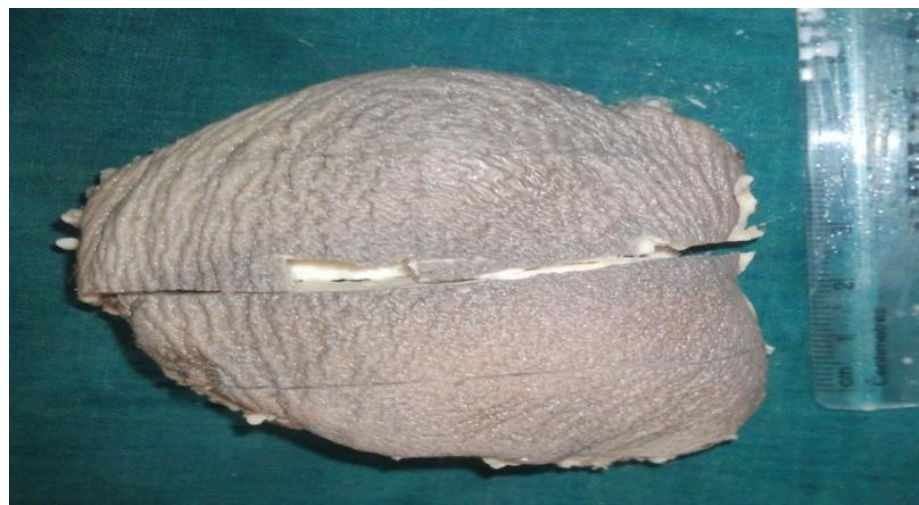

Figure1: Gross excised specimen showing a skin covered tissue mass.

Histopathological examination of the lesion confirmed the diagnosis of Fibrous hamartoma of infancy, the operative and the post operative period were uneventful and the child is on regular follow up.

\section{Gross pathology:}

\section{Histopathological Examination} $5 \times 4 \times 4 \mathrm{cms}$

A firm ivory white, unencapsulated, poorly circumscribed skin covered lobulated mass of dimensions:

The dissection was rubbery, fibrous and cut section revealed a gray white-glistening surface with irregular yellow fibrofatty areas. No areas of hemorrhage or necrosis noted.

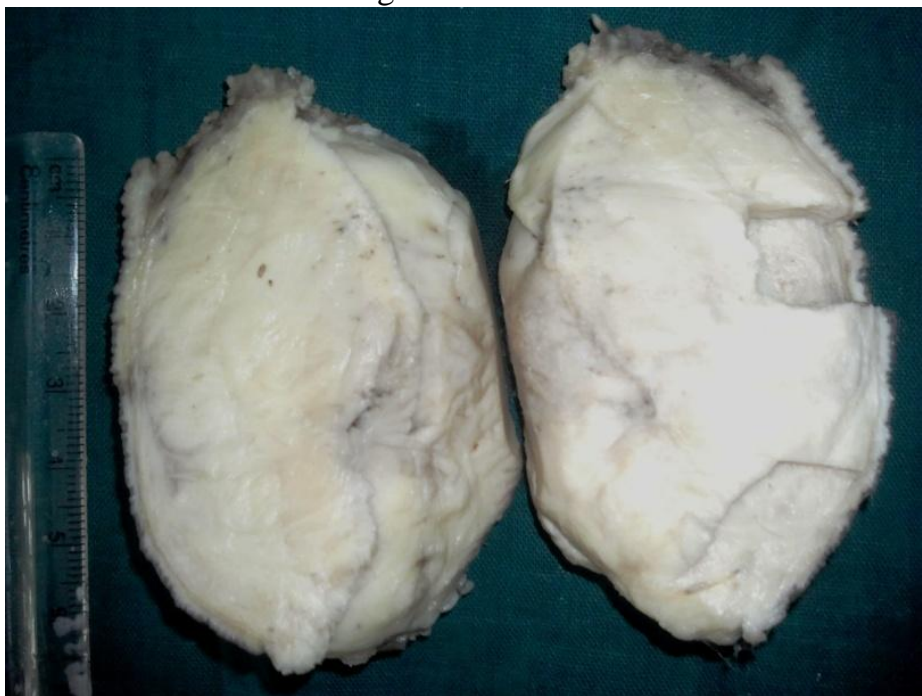

Figure2: Cut section of gross excised specimen showing solid glistening-white unencapsulated nature of tumor.

\section{Microscopic examination:}

Sections revealed thinned out stratified squamous epidermal lining. Underneath unencapsulated tumor tissue with a characteristic vague irregular triphasic organoid growth pattern consisting of-

1. Mature adipose tissue.

2. Intersecting trabeculae of hyalinized collagenous tissue.

3. Loosely textured areas of primitive mesenchymal tissue with scattered spindle to stellate shaped cells embedded in a mucopolysaccharide rich myxoid stroma/matrix.

No nuclear atypia or increased mitotic activity noted. No areas of hemorrhage or necrosis seen. 


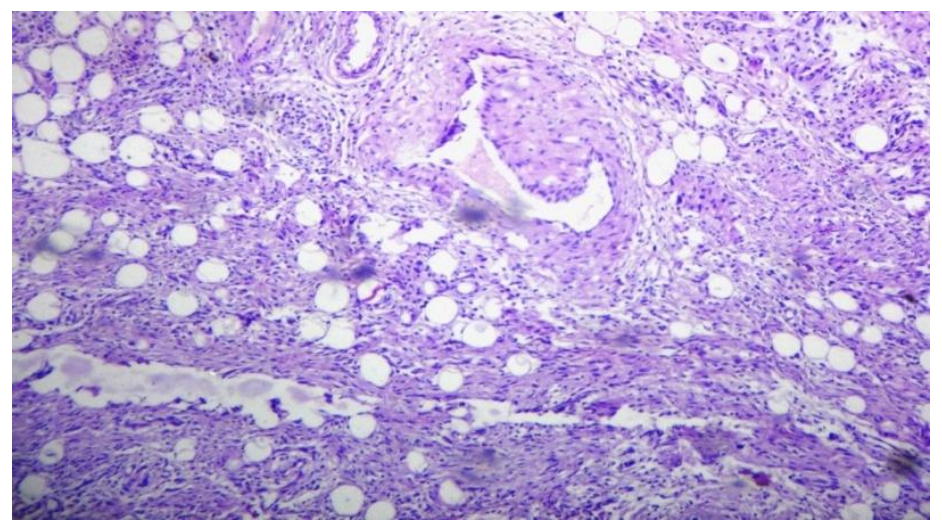

Figure3: Scattered adipocytes separated by bands of collagen. (H\&E, 04X)

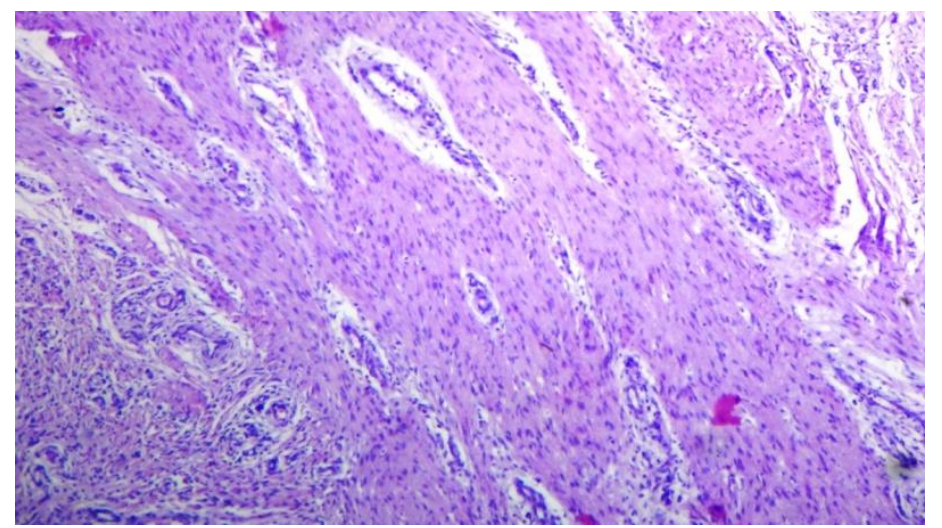

Figure4: Intersecting bands of hyalinized collagen. (H\&E stain, 10X)

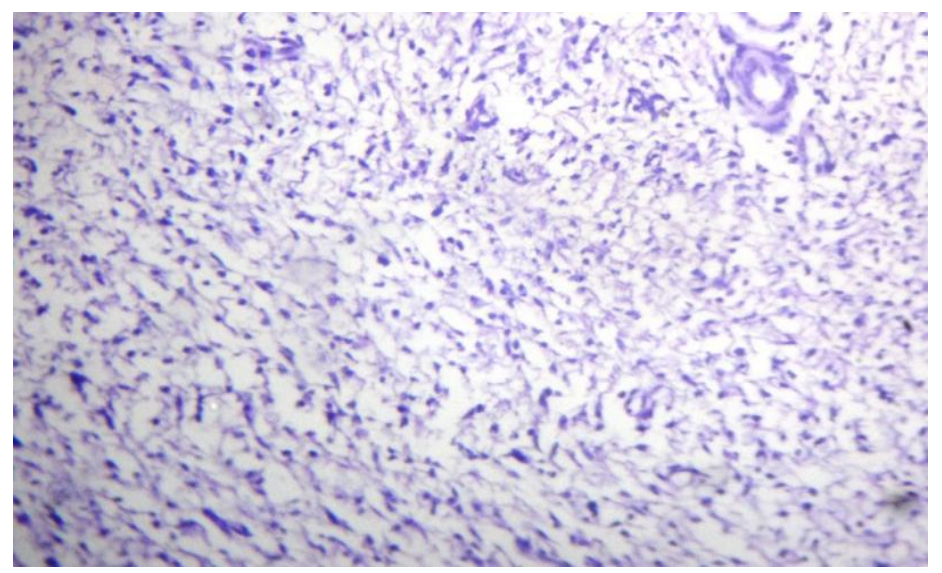

Figure5: Loosely textured primitive spindle and stellate cells. (H\&E stain, 10X)

The final histopathological diagnosis was Fibrous hamartoma of infancy.

\section{Discussion}

Fibrous hamartoma of infancy is an extremely rare, benign soft tissue neoplasms of uncertain histiogenesis with less than 200 cases reported in medical literature. ${ }^{[1]}$

This rare neoplasm was initially described by Reye in 1956 and suggested, it might be an aberrant reparative- regenerative process to an unknown injury or pathology rather than a true neoplasm. $\mathrm{He}$ hypothesized it to be of myofibroblastic origin and called it 'Subdermal fibrous tumor of childhood'. ${ }^{[2]}$

Enzinger in 1965; in his review of 30 cases termed the lesion as 'Fibrous hamartoma of Infancy' due to its organoid microscopic features and frequent occurrence at birth and infancy. ${ }^{[3]}$

WHO refers to it as a pediatric, benign, poorly delimited/circumscribed superficial soft tissue proliferation; characterized by triphasic organoid mixture of well defined intersecting trabeculae of dense fibrocollageous tissue, mature adipocytes and loosely textured areas of small primitive mesenchymal cells. ${ }^{[7]}$ 
It usually presents as a rapidly progressive painless, solitary tumor and more than $91 \%$ cases are reported within the first year of life and $15-20 \%$ of the cases have been described to be congenital. ${ }^{[4]}$

There is an overwhelming skew towards male affliction, with a male to female ratio of $2.4: 1 .{ }^{[1]}[5]$

Clinically the lesion is characterized by rapid progressive asymptomatic growth and most commonly have been reported to occur over the axilla, upper arm, shoulder, back, head and neck and sacral and inguinoscrotal regions. ${ }^{[6]}$

The tumors tend to be firm, non-tender, poorly circumscribed, with ill defined margins. The tumor can achieve great sizes ranging from $0.5-10 \mathrm{~cm}$ and in long standing cases more than $20 \mathrm{cms} .{ }^{[8]}$

Lesions are solid, painless, subcutaneous masses which may or may not be fixed to the deeper structures. Sometimes trophic changes may be seen in the overlying skin. There has been no evidence of familial or syndromic clustering of the cases. ${ }^{[1]}$

The histopathology is a kaleidoscopic mixture of 3 components i.e. thick intersecting bands of collagen, benign mature adipose tissue and loosely textured areas of primitive mesenchyme. The all 3 components arranged in an organoid pattern.

The rapid growth, size, fixation to deeper structures can be quite alarming both for the parents and the clinician. And it is pertinent not to confuse it with aggressive pediatric soft tissue neoplasms like rhabdomyosarcomas or undifferentiated sarcomas.

Lakshminarayanan $\mathrm{R}$ et al 2005 reported a reciprocal translocation, $\mathrm{t}(2 ; 3)(\mathrm{q} 31 ; \mathrm{q} 2)$ and a possible genomic loci on chromosome $2 \mathrm{q} 31$, coding the vitronectin receptor- $\alpha$ subunit of the integrin family. ${ }^{[9]}$

Rougemont et al 2006 showed a complex translocation $\mathrm{t}(6 ; 12 ; 8)(\mathrm{q} 25 ; \mathrm{q} 24.3 ; \mathrm{q} 13)$ in these lesions, thus validating the true neoplastic nature of the lesion. ${ }^{[10]}$

These irregular poorly delineated masses with tumor extensions in the surrounding tissue are a cause for tumor recurrence which may be seen in $10-16 \%$ of the cases. ${ }^{[4]}$

Carretto et al in 2006 in his evaluation of 18 cases of Fibrous hamartoma of infancy, concluded that treatment of choice for Fibrous hamartoma of infancy is wide local excision taking judicious margins with tissue reconstruction; and it is imperative not to over diagnose and over treat these benign lesions with radical and ablative procedures. ${ }^{[11]}$

\section{Conclusion}

Fibrous hamartoma of infancy is a rare benign lesion with typical cytomorphological features. FNAC, core biopsies can be misleading because of the triphasic, organoid nature of the lesion and so are the radiological features.

A strong clinical acumen coupled with prompt wide local excision and subsequent histopathological confirmation is in the best interest of the patient.

\section{References}

[1] Eyer de Jesus L, Schott GV, Jurema NR, Bilche GG, Penha M. Children's fibrous hamartoma: extensive injury involving brachial plexus. Case report.Acta Ortop Bras 2006;14:229-230.

[2] Reye RD. A consideration of certain subdermal fibromatous tumors of infancy.J Pathol. 1956; 72:149-54.

[3] Enzinger FM. Fibrous hamartoma of infancy. Cancer 1965;18:241-248

[4] DickeyGE, Sotelo-Avila C. Fibrous hamartoma of infancy: current review. Pediatr Dev Pathol. 1999; 2(3):236-43.

[5] Carretto E, Dall'Igna P, Alaggio R, Siracusa F, Granata C, Ferrari A, et al.Fibrous hamartoma of infancy: an Italian multiinstitutional experience. J Am Acad Dermatol 2006;54:800-803.

[6] Enzinger FM,Weiss SW:Fibrous proliferations of infancy and childhood.in soft tissue tumors. $2^{\text {nd }}$ ed.St.Louis,Missouri:The C.V Mosby Company,pp 164-168,1988.

[7] Hashimoto H. Fibrous hamartoma of infancy. In: World Health Organization Classification of Tumours. Pathology and Genetics of Tumours of Soft Tissue and Bone. Lyon: IARC Press; 2002. pp 58-59.

[8] Leticia del Carmen Baena-Ocampo, Luis Miguel Linares-Gonzalez, Norma Marin-Arriaga.Fibrous hamartoma of infancy.Case report.Cir Ciruj 2009;77:455-458.

[9] Lakshminarayanan R, Konia T, Welborn J. Fibrous hamartoma of infancy: a case report with associated cytogenetic findings. Arch Pathol Lab Med. 2005; 129(4):520-2.

[10] Rougemont AL, Fetni R, Murthy S, et al. A complex translocation (6;12;8) (q25;q24.3;q13) in a fibrous hamartoma of infancy. Cancer Genet Cytogenet. 2006;171(2):115-8.

[11] Carretto E, Dall'Igna P, Alaggio R, et al.Fibrous hamartoma of infancy: an Italian multi-institutional experience. J Am Acad Dermatol. 2006;54(5):800-3. 